\title{
An accurate millisecond timer for the Commodore 64 or 128
}

\author{
CARL A. HORMANN and JOSEPH D. ALLEN \\ University of Georgia, Athens, Georgia
}

\begin{abstract}
The use of the Commodore 64 or 128 as an accurate millisecond timer is discussed. BASIC and machine language programs are provided that allow the keyboard to be used as the response manipulandum. Modifications of the programs for use with a joystick or external switches are also discussed. The precision, flexibility, and low cost of these machines recommends their use as laboratory instruments.
\end{abstract}

Although timing routines with millisecond resolution are available for TRS-80 (Owings \& Fiedler, 1979) and 6500 series (Price, 1979) microcomputers, none are specifically written for the Commodore $64(\mathrm{C}-64)$ or the more recent Commodore $128(\mathrm{C}-128)$. The programs presented below transform the $\mathrm{C}-64$ or the $\mathrm{C}-128$, operating in either its 64 or 128 mode, into an accurate millisecond timer. Using these programs does not, however, limit the computer to timing applications. The programs are designed to serve as subroutines that end users may incorporate into programs of their own design, thus allowing increased flexibility.

These Commodore computers are easy to program and interface with external equipment, and the C-64, especially, offers considerable computing power at a relatively modest cost (the C-64, a monochrome monitor, and the 1541 disk drive can currently be purchased for under $\$ 400)$.

\section{DESCRIPTION OF TIMER}

\section{Timers Within the C-64}

The C-64 and C-128 are equipped with three timing sources, namely, a user-programmable time-of-day clock (resolution to .1 sec), a jiffy clock that counts the systemgenerated interrupts (resolution to $.16 \mathrm{sec}$ ), and four 2 byte counter/timers located on two 6526 Complex Interface Adapters (CIAs) (resolution to $1 \mu \mathrm{sec}$ ). The versatility of the C-64's and C-128's timers make the machines particularly useful as laboratory controllers.

\section{CIA Timers as Millisecond Timers}

The two 16-bit registers, referred to as timer A (TA) and timer $B(T B)$, which are located on CIA \#2, are free for use. They may be loaded with values between 0 and 65535 , and may be set to decrement at a rate determined

The authors wish to express their thanks to the University of Georgia Electronics Shop for the use of the Systron Donner Counter used to test the timer.

Requests for reprints and inquiries concerning the programs may be directed to either author at: Department of Psychology, University of Georgia, Athens, GA 30602. by the system clock, which is $1.022727 \mathrm{MHz}$. In order to obtain resolution in the millisecond range, TA is loaded with the value 1022 ( $\$ 3 F E$ ) and is started in its recycling mode by setting bits 0 and 4 in control register A (CRA). This configuration results in TA's underflowing 1000.3 times per second (a value as close to $1 \mathrm{msec}$ as can be achieved using binary registers). Since TB is counted down by TA underflows, initializing TB with 65535 (\$FFFF) permits elapsed times up to $1.5 \mathrm{~min}$ to be counted in milliseconds by complementing the value in TB, after TA has been stopped.

The millisecond timer was calibrated with a Systron Donner 1037 Series Counter (Model No. 1924). Using a sample rate of $1 \mathrm{sec}$, the timer was accurate to $\pm 0.05 \mathrm{msec}$.

\section{HARDWARE REQUIREMENTS}

The programs presented here are designed for use with both the C-64 and C-128 microcomputers. A disk drive (Commodore model 1541, 1571, or MSD SD1/2) and monitor (or television) are required accessories. Unless switches are used, no other hardware is needed.

\section{THE SOFTWARE}

\section{Overview}

The assembly language portion of the timer software was written to be located in the computer's cassette buffer (addresses 828-1019 in the C-64 or C-128 in the 64 mode; 2816-3007 in the C-128) but is fully relocatable to any convenient area of RAM. The program may be entered using any full-featured assembler, such as PAL64 (Proline Software); using an extended monitor, such as super- or micromon; or using the BASIC Loader program listed in Figure 1.

ML64 TIMER.LDR. When entering the BASIC loader listing in Figure 1, the user should treat the words within the brackets as cursor controls, not as literals. When run, this program sets up the machine code portion of the timer program in locations 828-928 of the cassette buffer and operates correctly on the C-64 or the C-128 in its 64 mode. This program should be loaded and run first. If the $\mathrm{C}$ - 
10 REM BASIC LOADER FOR C-64 ML TIMER

20 FOR $I=828$ TO $928:$ READ $X: P O K E I, X: T=T+X: N E X T$

30 IF T<>13377 THEN PRINT"<CLR $\rangle\langle$ DWN $\rangle\langle$ DWN $\rangle\langle$ DWN $\rangle\langle$ DWN $\rangle$ ENTRY ERROR: CHECK YOUR DATA

VALUES" : STOP

40 END

1000 DATA $169,127,162,254,160,3,141,13$

1001 DATA $221,142,4,221,140,5,221,169$

1002 DATA $255,141,0,221,141,7,221,160$

1003 DATA $81,140,15,221,96,120,169,1$

1004 DATA $141,25,208,173,17,208,41,127$

1005 DATA $141,17,208,173,25,208,41,1$

1006 DATA $240,249,173,17,208,9,16,141$

1007 DATA $17,208,162,17,142,14,221,32$

1008 DATA $159,255,166,203,224,4,240,4$

1009 DATA $224,3,208,243,169,0,141,14$

1010 DATA $221,134,252,173,6,221,73,255$

1011 DATA $133,250,173,7,221,73,255,133$

1012 DATA $251,88,184,80,170$

Figure 1. ML64 TIMER.LDR: Listing of BASIC loader program for loading the machine language timer program. (Words in brackets should be interpreted as cursor control commands.)

128 is used in its regular mode, then the following lines must be substituted:

20 BANK 15: FOR I =2816 TO 2916: READ X: POKE I,X: $\mathrm{T}=\mathrm{T}+\mathrm{X}: \mathrm{NEXT}$

1008 DATA $159,255,166,212,224,4,240,4$

TIMER64.BAS. The program listed in Figure 2 sets up and measures the time in milliseconds required for a subject to assign words displayed serially on the monitor to one of two mutually exclusive cognitive categories, mammalian or nonmammalian. The two function keys Fl and F7 were arbitrarily selected to record the choice. Ten trials are presented; then the subject's reaction times and associated response choices are printed to the screen or a printer and then saved as the sequence file DATAFILE on a disk.

Stimulus displays to the screen can present a complex problem for accurate timing, since the screen is refreshed or updated only 60 times each second (Reed, 1979; Kallman, 1986). Thus the time between writing the stimulus to screen memory and its subsequent display to the screen can vary up to $16.67 \mathrm{msec}$, which, with a base reaction time of $250 \mathrm{msec}$, constitutes a $6.7 \%$ error in measurement. This problem can be solved by synchronizing the display driver with the monitor's scanning circuit. Each sweep requires a little more than $1 \mathrm{msec}$ and scans the monitor with 256 rasters, with rasters 51 and 250 representing the top and bottom visible lines on the screen. Fortunately, the C-64 or C-128 continuously monitors the screen refresh, and the current raster can be accessed at any time from the raster register (location 53266) of the VIC chip. In fact, a value written to this register is latched and may be compared with the raster scan. When the current raster matches the value in the latch, a flag in the IRQ Sense Register (53273) is set. Thus a screen display can be timed to coincide with the proximity of the raster. For example, if one wishes to display the stimulus on row 12 of the screen, a value of 138 (11 rows $\times 8$ raster lines per row +50 ) must be POKEd into the raster register, and the raster IRQ flag will be set when the raster reaches the beginning of row 12 .
Writing the display driver routine in BASIC affords the ease and simplicity of managing stimulus displays as character string arrays; however, printing a complex display would be too slow to synchronize reliably with the raster scan. A machine language (ML) display driver greatly speeds up display production and permits synchronization, but at the cost of setting up and managing complicated stimulus tables. A superior method is to use display blanking, a feature supported by the VIC chip. Clearing the display enable bit (bit 4) of register 53265 blanks the screen with the color of its border, allowing elaborate displays to be manufactured in BASIC and set up ahead of time. A machine language routine can then be used to instantly unblank the screen when the raster is in proximity with the first row of the display and then quickly jump to start the timer that measures reaction time.

This is the method used by the demonstration program in Figure 2. Program lines 10-90 dimension stimulus and data arrays, set the screen and border color to light gray, set the raster register to detect the 138th raster, and call the initializing routine of the ML timer program that configures the millisecond timer in the CIA chip. The userwritten portion of the program begins with line 100 . A stimulus word is randomly selected and printed in row 12 . Important lines include line 240, which blanks the screen prior to filling screen memory with the stimulus display, and lines 260-290, which compute a random intertrial delay that is reset by random key holding. Entry to the ML display/timer program is gained in line 340 only after the keyboard buffer is emptied of stray characters that might be decoded by the ML program as 0-msec reaction times.

Upon return to BASIC, reaction times are extracted from locations 250-251 and assigned to a data array. Also, the key selected, $F 1$ or F7, is returned in location 252 , decoded, and assigned to an array. After 10 trials, the operator is given, in lines $420-500$, the option of either printing the formatted reaction time and response choice data either to the screen or to a printer. Finally, lines 520-600 save the data to a sequence file named DATAFILE.

For those using a C-128 in 128 mode, the SYS address 


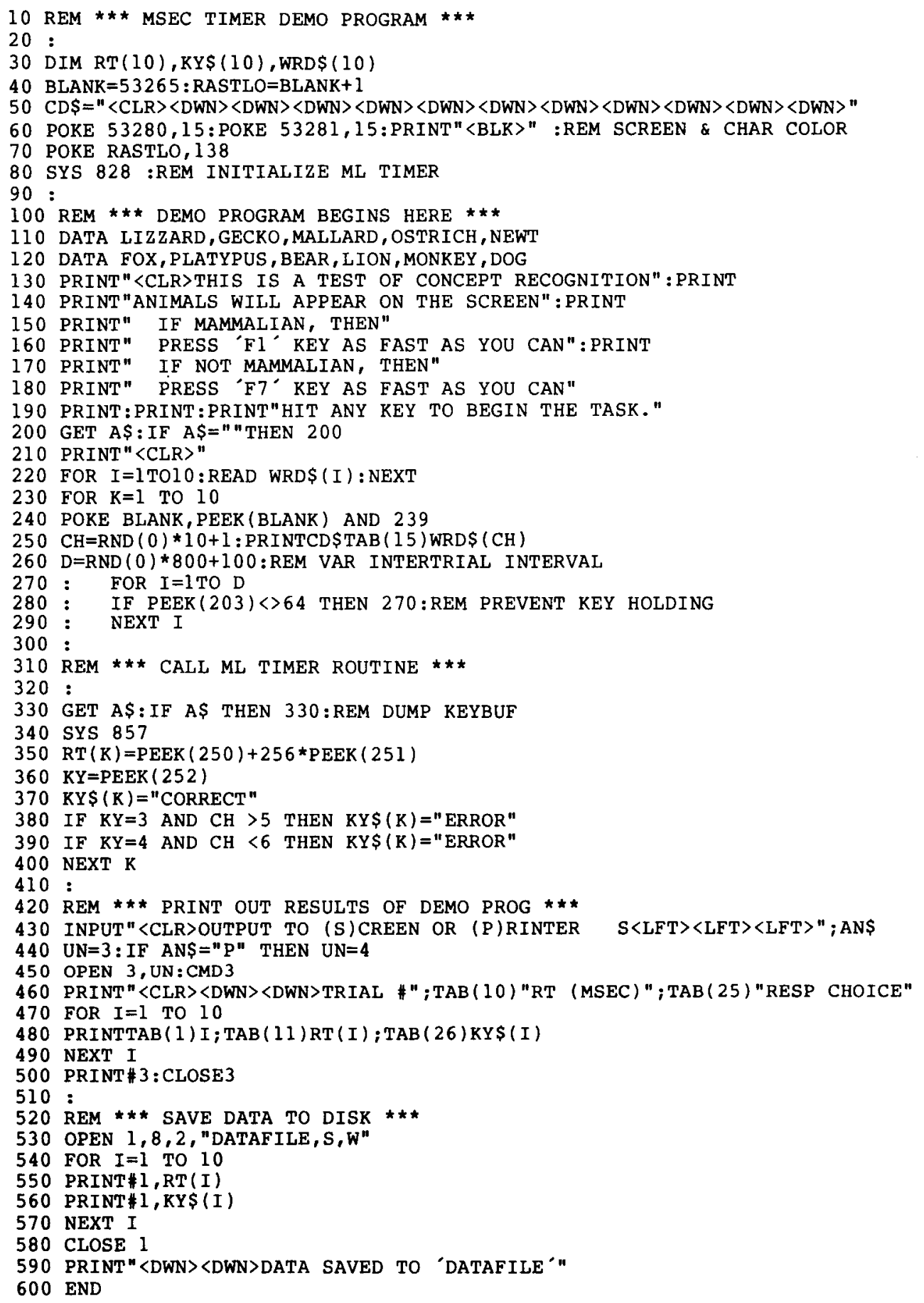

Figure 2. TIMER64.BAS: Listing of BASIC controller/demonstration program. (Words in brackets should be interpreted as cursor control commands.)

in line 80 must be changed from 828 to 2816 and SYS 857 in line 340 must be changed to SYS 2845 to correspond to a relocated cassette buffer. Also, line 280 should be modified as follows:

$$
280 \text { : IF PEEK(212) }<>88 \text { THEN } 270 .
$$

The program will now run in the C-128's default mode using the 40-column screen only. A different chip altogether, the 8563 Video Controller, operates the C-128's 80-column screen. Although fully capable of synchroniz- ing displays, the Video Controller requires different and more sophisticated driver routines than are required by either the 6566 or the 8564 VIC II chips that control the 40-column screens in the C-64 and C-128.

ML TIMER64.SRC. The source code for the ML timer program was written using the PAL64 assembler (Proline Software) and appears in Figure 3. As listed, it is designed for use with the keyboard (i.e., pressing either function key F1 or F7 stops the timer) and operates correctly on either the C-64 or the C-128 in 64 mode. 
20: $\quad 033 \mathrm{C}$

. OPT P4
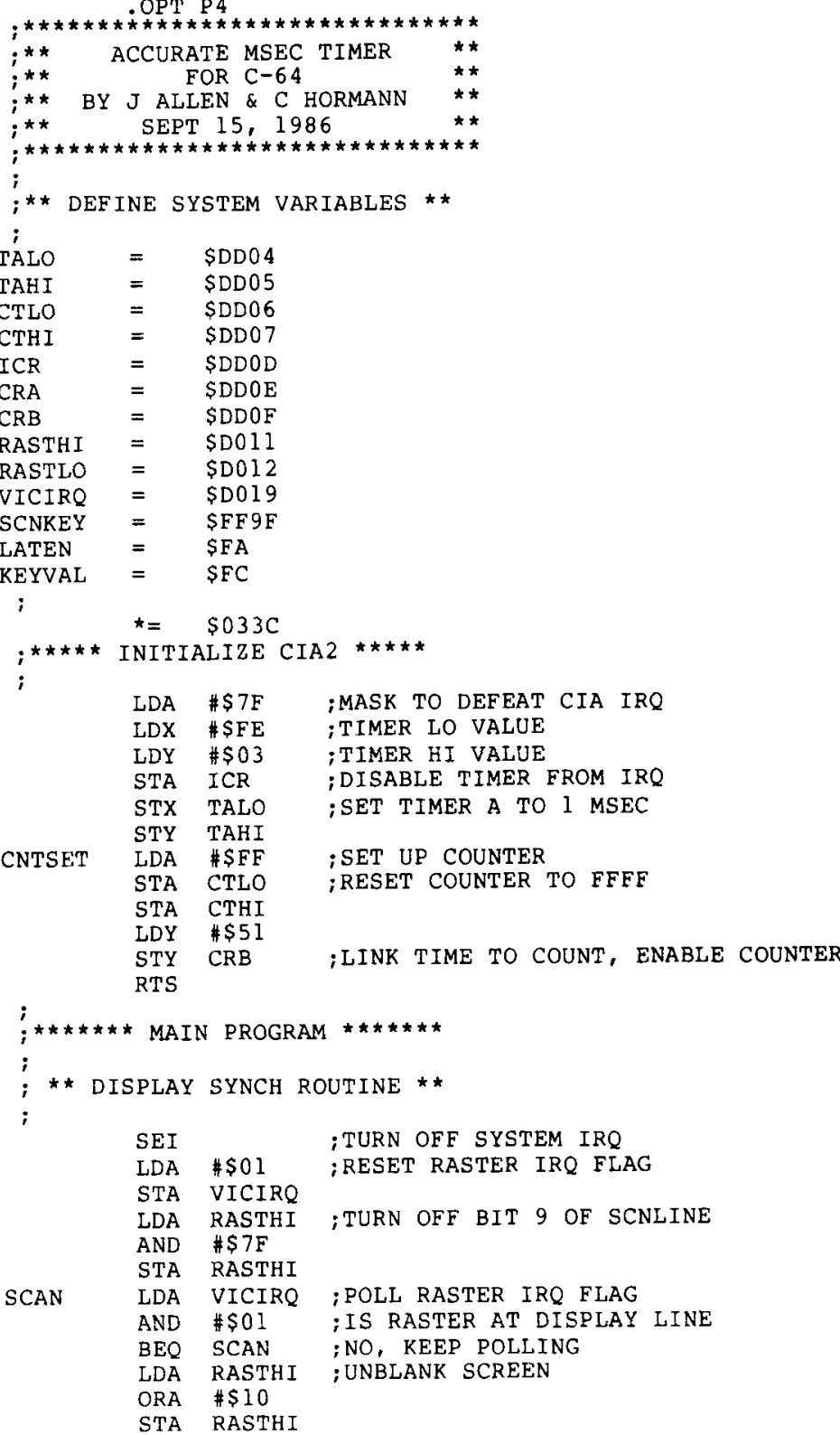

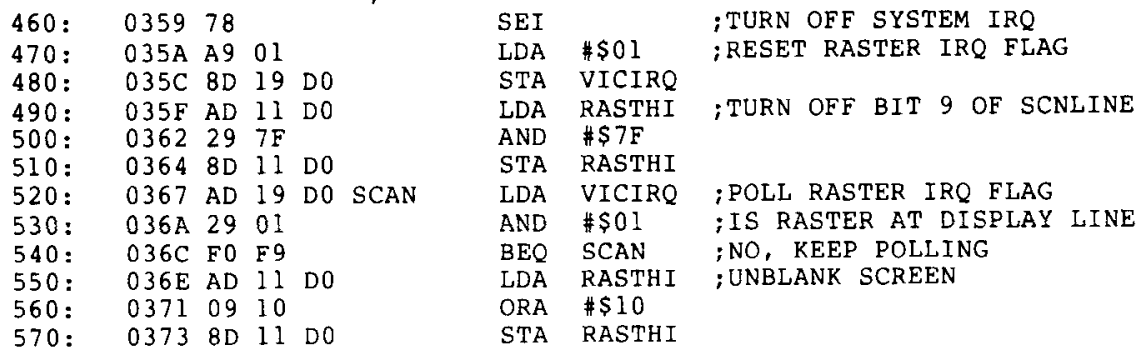

; * TIME LATENCY TO RESPOND **

610: 0376 A2 $11 \quad$ LDX \#\$11 ;SET \& START TIMER A

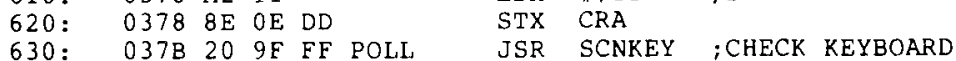

640: $037 \mathrm{E} \mathrm{A6} \mathrm{CB} \mathrm{LDX} \mathrm{SCB} \mathrm{:CHECK} \mathrm{KEYCODES} \mathrm{FOR} \mathrm{Fl} \mathrm{OR} \mathrm{F7}$

650: 0380 EO $04 \quad$ CPX \#\$O4 ;IS FI' PRESSED

660: 0382 F0 04 BEQ STOPCLOK ; YES

670: 0384 E0 $03 \quad$ CPX \#\$03 ;IS 'F7' PRESSED

680: 0386 DO F3 BNE POLL ;NEITHER, SO POLL AGAIN

690: 0388 A9 00 STOPCLOK LDA \#\$00; STOP THE CLOCK

$700: 038 \mathrm{~A} 8 \mathrm{D} 0 \mathrm{E}$ DD

$710: 038 \mathrm{D} 86 \mathrm{FC}$

720: $038 \mathrm{~F}$ AD 06 DD

730: $039249 \mathrm{FF}$

$740: 039485 \mathrm{FA}$

$750: 0396$ AD 07 DD

760: $039949 \mathrm{EF}$

$770: \quad 039 \mathrm{~B} 85 \mathrm{FB}$

$780: \quad 039058$

$790: \quad 039 \mathrm{E} \quad \mathrm{B} 8$

$800: \quad 039 \mathrm{~F} 50 \mathrm{AA}$

STA CRA

STX KEYVAL ; RECORD WHICH KEY PRESSED

LDA CTLO ; GET COUNTER LO VALUE

EOR \#\$FF ; COMPLEMENT FOR LATENCY\&

STA LATEN ; STORE IT

IDA CTHI ; SAME FOR COUNTER HI BYTE

EOR \#SFF

STA LATEN+1

CLI $\quad$;RETURN IRQS TO SYSTEM

Figure 3. ML TIMER64.SRC: Listing of millisecond timer and display synchronization source code for use with the keyboard. 


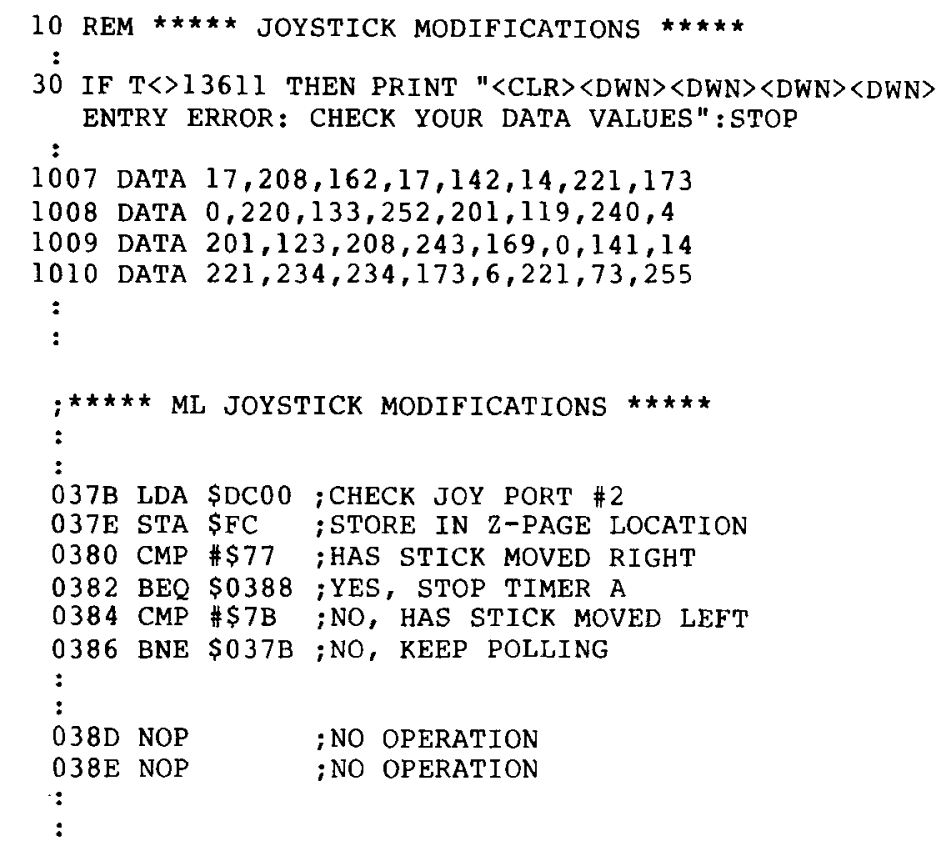

Figure 4. Modifications to MI64 TIMER.LDR and ML TIMER64.SRC necessary for use with a joystick. (Those lines of the original programs that do not change are indicated by colons.)

The first portion of the program (lines 290-400) configures the millisecond timer in CIA \#2. It disables timergenerated interrupts, loads the appropriate countdown value ( $\$ 3 \mathrm{FE}$ ) into timer $A$, sets timer $B$ to count timer $A$ underflows, and resets timer B to \$FFFF. This initialization routine is called at the beginning of the BASIC controller program and need only be executed once at startup. The main portion of the program is broken into two parts, the display synchronization and the timer routines. Upon entry to the display synch routine (lines 460-570), the system IRQ is turned off and any raster-generated flag is cleared before the IRQ register is polled for a match between the raster location and the scan line (138) POKEd into the raster register by the BASIC program. The match will occur when the raster scan is beginning line 12 , which contains the hidden display. The program drops through the polling loop to unblank the screen, then executes the timing routine contained in lines $610-800$.

The timer portion starts timer $A$ and then polls the keyboard, decoding for F1 and F7 keypresses, using a kernal routine that completes a keyboard scan in less than 1 msec. The F1 and F7 keys are arbitrary choices and may be replaced with any keys that the investigator desires (see Timer Modifications below). Once either keypress is detected, timer A is stopped and the key code of the depressed key is temporarily stored in a zero-page memory location. The values in timer $B$ are then complemented and stored in a 2-byte zero-page location (250-251) for later recovery and conversion by BASIC into a reaction time. The program then branches up to the latter part of the initialization routine, where timer B is reset to \$FFFF and control is returned to BASIC.

To use this program in the C-128's default mode, line 640 must be changed to read:

\section{LDX \#\$D4}

Assembly should begin at $\$ 0 \mathrm{~B} 00$, the start of the C-128's cassette buffer.

\section{Timer Modifications}

The BASIC and ML programs may be easily modified so that either a joystick or external switches may serve

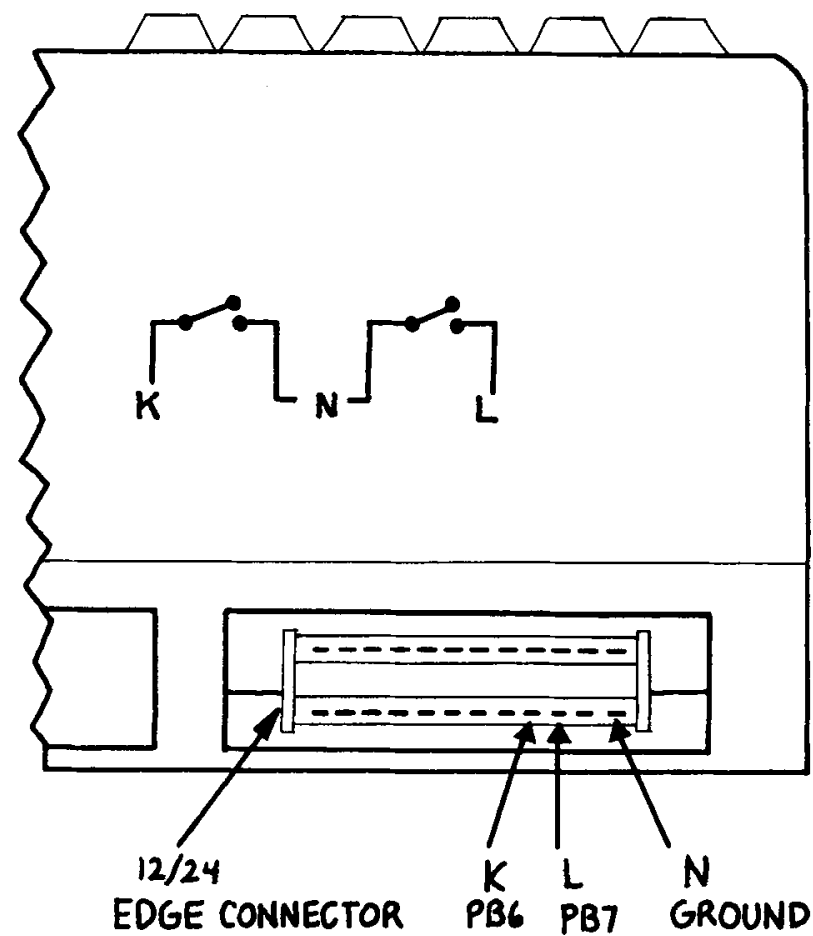

Figure 5. Illustration of the user port with an edge connector attached and associated schematic diagram for switch interfacing. 


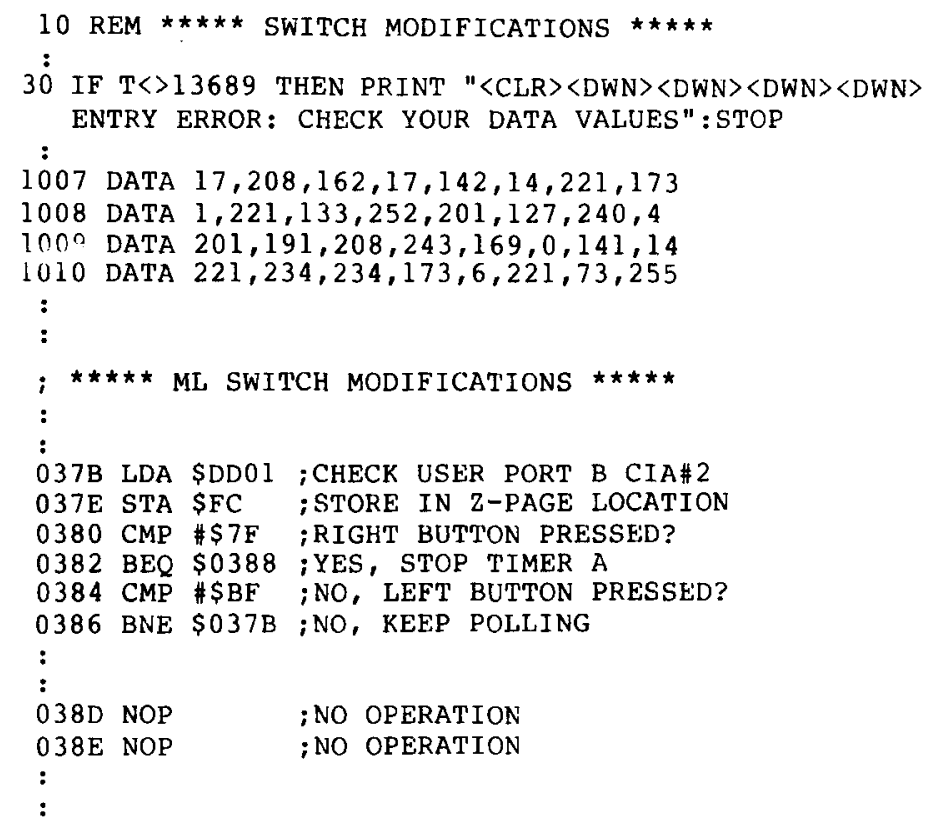

Figure 6. Modifications to ML64 TIMER.LDR and ML TIMER64.SRC necessary for use with external switches. (Those lines of the original programs that do not change are indicated by colons.)

as the response manipulandum. These modifications increase the versatility of the timer and obviate the need for having the computer in front of the participants.

Use with a joystick. In order to use the timer program with a joystick (attached to Joy Port \#2), the polling loop in the main ML program must be modified. Lines 630-680 must be deleted and a different set of code inserted (see Figure 4 for the necessary BASIC and ML source code modifications). Once the modifications have been completed, the program will detect left and right movements of a joystick.

Use with an external switch. Use of this program with an external switch necessitates the purchase of SPST momentary switches (e.g., Radio Shack No. 275-1547), wire, and a $12 / 24$ edge connector $(0.156$ in off center; a suitable connector is model $12 / 24 \mathrm{SE}$, supplied by Jameco Electronics, 1355 Shoreway Rd., Belmont, CA 94002). Figure 5 is the schematic diagram for interfacing the switches with either the C-64 or the C-128 computer. One contact on each of the switches should be soldered (using enough wire to allow for freedom of movement) to pin $\mathbf{N}$ (ground) of the edge connector. The remaining contact of the switch, to be located on the right, is hooked to pin $\mathrm{L}$ ( $\mathrm{PB} 7$ ), and the remaining contact of the switch on the left is hooked to pin K (PB6).

Once the soldering has been completed, the connector should be attached to the user port of the C-64 or C-128, with the lettered side on the bottom (attach and remove the connector only when the machine is off). When either switch is pressed, the input/output (I/O) line to which it is attached is grounded. Further information about the user port may be obtained by consulting West (1985) and De Jong (1984).

The ML program, for use with the switches, operates by checking the user port $\mathrm{I} / \mathrm{O}$ lines for the presence of grounded lines. In order for the program to accomplish this, lines 630-680 of the ML TIMER64.SRC program must be deleted and a different set of code inserted (see Figure 6 for the necessary BASIC and ML source code modifications).

Modifications to ML TIMER64.SRC. Some users may wish to change the keys used to enter a response. This may be accomplished by determining the keyboard buffer (not ASCII) code of the desired keys (values may be found in West [1985, p. 161]) and substituting their hexadecimal equivalents for the values in lines 650 and 670.

The values may also be changed using the BASIC loader program. Once the values of the keys are determined, the (decimal) value of one should be used in place of the first 4 in line 1008 of the BASIC program, and the value of the other should be substituted for 3 in line 1009 .

\section{REFERENCES}

DE JoNG, M. L. (1984). Assembly language programming with the Commodore 64. Bowie, MD: Brady Communications.

Kallman, H. J. (1986). A Commodore 64-based experimental psychology laboratory. Behavior Research Methods, Instruments, \& Computers, 18, 222-227.

OWINGS, R. A., FIEDLER, C. H. (1979). Measuring reaction time with millisecond accuracy using the TRS-80 microcomputer. Behavior Research Methods \& Instrumentation, 11, 589-591.

Price, J. M. (1979). Software timing for 6500 series microcomputers. Behavior Research Methods \& Instrumentation, 11, 568-571

ReED, A. V. (1979). Microcomputer display timing: Problems and solutions. Behavior Research Methods \& Instrumentation, 11, 572-576.

WEST, R. C. (1985). Programming the Commodore 64: The definitive guide. Greensboro, NC: Compute! Publications. 